\title{
CT-Guided Percutaneous Transthoracic Localization of Pulmonary Nodules Prior to Video-Assisted Thoracoscopic Surgery Using Barium Suspension
}

\author{
Nyoung Keun Lee, $M D^{1}$, Chang Min Park, $M D^{1}$, Chang Hyun Kang, $M D^{2}$, Yoon Kyung Jeon, $M D^{3}$, \\ Ji Yung Choo, MD' ${ }^{1}$, Hyun-Ju Lee, $M D^{1}$, Jin Mo Goo, MD \\ ${ }^{1}$ Department of Radiology, Seoul National University College of Medicine, and Institute of Radiation Medicine, Seoul National University Medical \\ Research Center, Seoul 110-744, Korea; Departments of ${ }^{2}$ Thoracic and Cardiovascular Surgery and ${ }^{3}$ Pathology, Seoul National University College of \\ Medicine, Seoul 110-744, Korea
}

Objective: To describe our initial experience with CT-guided percutaneous barium marking for the localization of small pulmonary nodules prior to video-assisted thoracoscopic surgery (VATS).

Materials and Methods: From October 2010 to April 2011, 10 consecutive patients (4 men and 6 women; mean age, 60 years) underwent CT-guided percutaneous barium marking for the localization of 10 small pulmonary nodules (mean size, $7.6 \mathrm{~mm}$; range, 3-14 mm): 6 pure ground-glass nodules, 3 part-solid nodules, and 1 solid nodule. A $140 \%$ barium sulfate suspension (mean amount, $0.2 \mathrm{~mL}$; range, $0.15-0.25 \mathrm{~mL}$ ) was injected around the nodules with a 21-gauge needle. The technical details, surgical findings and pathologic features associated with barium localizations were evaluated.

Results: All nodules were marked within $3 \mathrm{~mm}$ (mean distance, $1.1 \mathrm{~mm}$; range, 0-3 mm) from the barium ball (mean diameter, $9.6 \mathrm{~mm}$; range, $8-16 \mathrm{~mm}$ ) formed by the injected barium suspension. Pneumothorax occurred in two cases, for which one needed aspiration. However, there were no other complications. All barium balls were palpable during VATS and visible on intraoperative fluoroscopy, and were completely resected. Both the whitish barium balls and target nodules were identifiable in the frozen specimens. Pathology revealed one invasive adenocarcinoma, five adenocarcinoma-in-situ, two atypical adenomatous hyperplasias, and two benign lesions. In all cases, there were acute inflammations around the barium balls which did not hamper the histological diagnosis of the nodules.

Conclusion: CT-guided percutaneous barium marking can be an effective, convenient and safe pre-operative localization procedure prior to VATS, enabling accurate resection and diagnosis of small or faint pulmonary nodules.

Index terms: CT; Localization; Percutaneous; Lung; Nodule; Barium

Received March 23, 2012; accepted after revision May 2, 2012. This study was supported by the Research Grant Number CB-201102-01 of the Korean Foundation for Cancer Research.

Corresponding author: Chang Min Park, MD, Department of Radiology, Seoul National University College of Medicine, and Institute of Radiation Medicine, Seoul National University Medical Research Center, 101 Daehak-ro, Jongno-gu, Seoul 110-744, Korea. - Tel: (822) 2072-0367 - Fax: (822) 743-7418

- E-mail: cmpark@radiol.snu.ac.kr

This is an Open Access article distributed under the terms of the Creative Commons Attribution Non-Commercial License (http://creativecommons.org/licenses/by-nc/3.0) which permits unrestricted non-commercial use, distribution, and reproduction in any medium, provided the original work is properly cited.

\section{INTRODUCTION}

As CT use becomes widespread in daily clinical practice and lung cancer screening settings, we have increasingly encountered small or faint, albeit clinically significant pulmonary lesions on $\mathrm{CT}(1,2)$. According to the ELCAP trial, $55.5 \%$ of detected lung cancers were small nodules of $10 \mathrm{~mm}$ or smaller (3), and $51.7 \%$ of detected lung cancers found during baseline screening were ground-glass nodules (GGNs), most of which are typically faint or invisible on plain radiographs (4). The need for accurate early diagnosis of these small or faint nodules with high suspicion of 
malignancy is evident. Unfortunately diagnosis of these small or faint lung nodules has remained a challenge with dedicated CT, FDG-PET/CT or even image-guided percutaneous biopsy.

In order to diagnose and treat such small or faint lung nodules with high clinical suspicions of malignancy, videoassisted thoracoscopic surgery (VATS), a less invasive type of thoracic surgery, has recently been adopted (5). However, a problem often encountered during VATS of these lesions was localization of the target nodule, which depended on its position, size, and characteristics such as nodule consistency. Further, when small nodules were located 1 or $2 \mathrm{~cm}$ below the pleura or further, it was difficult for the surgeon to determine their exact locations (6).

In this context, preoperative nodule localization is of critical importance in the accurate resection of these lesions. Today, metallic hook wire localization under CT guidance is the most widely used method for this purpose (7). However, this presents disadvantages. One disadvantage of metallic hook wire localization is the possibility of wire dislodgement. Therefore it is best performed immediately prior to the operation (7). Also, pneumothorax due to pleural injury caused by the wire and patients' complaints of discomfort from the hook wire in the chest wall have been documented $(8,9)$.

In contrast, a localization technique using barium suspension can be much simpler to perform with no risk of dislodgement, allowing the patient to move freely prior to the operation. Furthermore, the barium ball shows as white visually, is radiopaque on fluoroscopy and can be palpable during the operation, making detection more accurate and easier (10-13). With this technique the target lesions can be much more accurately localized and resected.

There have been several published reports dealing with barium marking in the literature (10-13), although the localization procedures were performed using bronchoscopy. To our knowledge, there have been no reports on CT-guided percutaneous barium localization including procedure details and the pathologic findings associated with this method. Thus, the purpose of our study was to describe our initial experience with CT-guided barium marking for localization of small pulmonary nodules prior to VATS.

\section{MATERIALS AND METHODS}

Our Institutional Review Board approved this retrospective study with waiver of informed consent for inclusion into this study. Written informed consent for the use of CTguided percutaneous localization using barium sulfate was obtained from all patients after discussion of all related risks and benefits.

\section{Patients and Nodules Characteristics}

From October 2010 to April 2011, CT-guided percutaneous barium localizations were performed for 10 pulmonary nodules in 10 consecutive patients ( 4 men and 6 women; mean age, 60 years; range, 44-72 years). Nodules were detected on lung cancer screening $\mathrm{CT}$ in 5 patients and 2 lesions were incidentally detected on CT taken due to symptoms of cough or sputum. In three patients, nodules were found during routine follow-up after treatment of an extrathoracic malignancy; two patients had a history of colon cancer, and one had a history breast cancer within 4 years (mean, 27 months; range, 13-42 months). Three were former smokers, and one was a current smoker. The mean number of pack-years was 16.5 (range, 5-26 pack-years). Characteristics of the nodules are summarized in Table 1.

\section{Procedural Details of Barium Localizations}

All CT-guided percutaneous barium localizations were performed on the day or one day prior to VATS. Before the localization procedure, contrast-enhanced chest CT images were carefully reviewed to decide the most appropriate route to the target nodules for successful marking and lowest probability of complications. Procedural details of barium localizations performed in our study follow:

1) Each patient was placed on a CT table in a suitable position (supine or prone). Then, after placing a radiopaque grid made of 0.032-inch guide-wire mesh on the intended region of the chest wall, a preprocedural CT scan was performed (Fig. 1A).

\section{Table 1. Characteristics of Nodules}

\begin{tabular}{ll}
\hline Maximum transverse diameter $(\mathrm{mm})^{*}$ & $7.6(3-14)$ \\
Distance from the pleura $(\mathrm{mm})^{*}$ & $8.4(0-23)$ \\
Location & 4 \\
RUL & 4 \\
RLL & 2 \\
\hline LUL & \\
Nodule feature & 1 \\
Solid nodule & 6 \\
Pure GGN & 3 \\
\hline Part-solid nodule
\end{tabular}

Note. - * Data are means and range in parenthesis. GGN $=$ groundglass nodule, $\mathrm{LUL}=$ left upper lobe, $\mathrm{RLL}=$ right lower lobe, $\mathrm{RUL}=$ right upper lobe 
2) Based on the preprocedural CT images, the skin entry site, exact intended site of barium injection and needle route to the target were determined. The barium injection was to be placed within $1 \mathrm{~cm}$ of the nodule. After local anesthesia of the chest wall using $2 \%$ lidocaine, a 21-gauge needle was introduced to the planned barium injection site using sequential CT image guidance to guide the path.

3) If the needle tip was accurately placed at the planned injection site, the inner stylet of the needle was withdrawn and a 140\% (weight/volume) barium sulfate suspension (Solotop Solution 140; Tae Joon Pharmaceuticals, Seoul, Korea) was injected through the needle. The average instilled volume of barium was $0.2 \mathrm{~mL}$ (range, $0.15-0.25 \mathrm{~mL}$ ).

4) Post-procedural CT was performed to identify the exact location and characteristics of the injected barium ball, and the presence of procedure-related complications (Fig. 1B). Blood pressure, breathing rate, and arterial oxygen saturation $\left(\mathrm{SaO}_{2}\right)$ of all patients were monitored throughout the procedure. The barium suspension hardened into a barium ball allowing it to be palpable.

\section{Data Analysis}

\section{Procedure Record and Radiation Dose}

One author (C.M.P. with 7 years of experience in imageguided thoracic intervention) performed and recorded the procedures and amount of radiation dose during barium localization. Procedural records included the size of the barium ball, distance between the barium ball and nodules, total procedure time, procedure-related complications and time interval between the localization procedure and the operation (Fig. 1C).

\section{Surgical Findings (Fig. 1C)}

One author (C.H.K. with 10 years of experience in thoracic surgery) recorded all surgical findings including the palpability/detectability of the barium ball during VATS, visibility of the radiopaque barium ball under intraoperative C-arm fluoroscopy, and successive resections of the barium ball and target nodule.

\section{Analysis of Histological Features Associated with Barium Markings}

All pathologic slices were reviewed and diagnosed by an experienced pathologist (Y.K.J. with 8 years of experience in pathology). All lesions were evaluated in terms of the pathologic diagnosis of the target nodule and spatial relationship between the barium ball and target nodule. The pathologic findings of lung parenchyma in the barium injection site and the influence of the barium on the definitive pathologic diagnosis of the target nodule were also evaluated (Fig. 1D).

\section{RESULTS}

All target nodules were marked within $3 \mathrm{~mm}$ distance (mean distance, $1.1 \mathrm{~mm}$; range, 0-3 $\mathrm{mm}$ ) from the injected barium sulfate suspension, which formed round or oval shaped balls, using the barium localization method (Figs. $2,3)$. On CT scans, the barium balls were placed 2-3 mm from the nodule in 5 patients and were superimposed on the nodules in the remaining 5 patients. Table 2 shows the procedure details of barium localization. Pneumothorax occurred in 2 patients, for which one needed aspiration. Other than these 2 patients, no complications occurred during or after this localization procedure.

All VATS procedures were performed 2 to 24 hours (mean, 13.2 hours) after barium localization. The surgeons were able to palpate the barium balls during VATS $(n=10)$ and the radiopaque barium balls were able to be indentified in all patients under intraoperative fluoroscopy $(n=10)$ (Figs. 1C, 2C, 3C). Under fluoroscopy, all radiopaque barium balls and target nodules near the barium balls were successfully resected with sufficient surgical margins. In all patients, the barium balls were identified as whitish nodules in the surgical specimens with both the barium balls and target nodules able to be identified in the frozen specimens. Pathologically, all nodules were definitively diagnosed (Table 3). The 10 target nodules were confirmed as invasive adenocarcinoma $(n=1)$, adenocarcinoma-in-situ $(n=5)$, atypical adenomatous hyperplasias $(n=2)$, and benign lesions $(n=2)$. On histopathologic examination, a nodule

\section{Table 2. Procedural Details of Barium Localization}

\begin{tabular}{lc}
\hline Total procedure duration (mins) & 25.6 (range; 15-48) \\
Diameter of barium balls (mm)* & 9.6 (range; $7.5-12)$ \\
Effective dose (mSv) & 1.56 (range; 0.76-2.34) \\
\hline
\end{tabular}

Note._ ${ }^{*}$ Data are means and range in parentheses.

Table 3. Histological Diagnosis of Nodules

\begin{tabular}{ll}
\hline Invasive adenocarcinoma & 1 \\
Adenocarcinoma-in-situ & 5 \\
Atypical adenomatous hyperplasia & 2 \\
Chronic granulomatous inflammation & 1 \\
Intrapulmonary lymph node & 1 \\
\hline
\end{tabular}



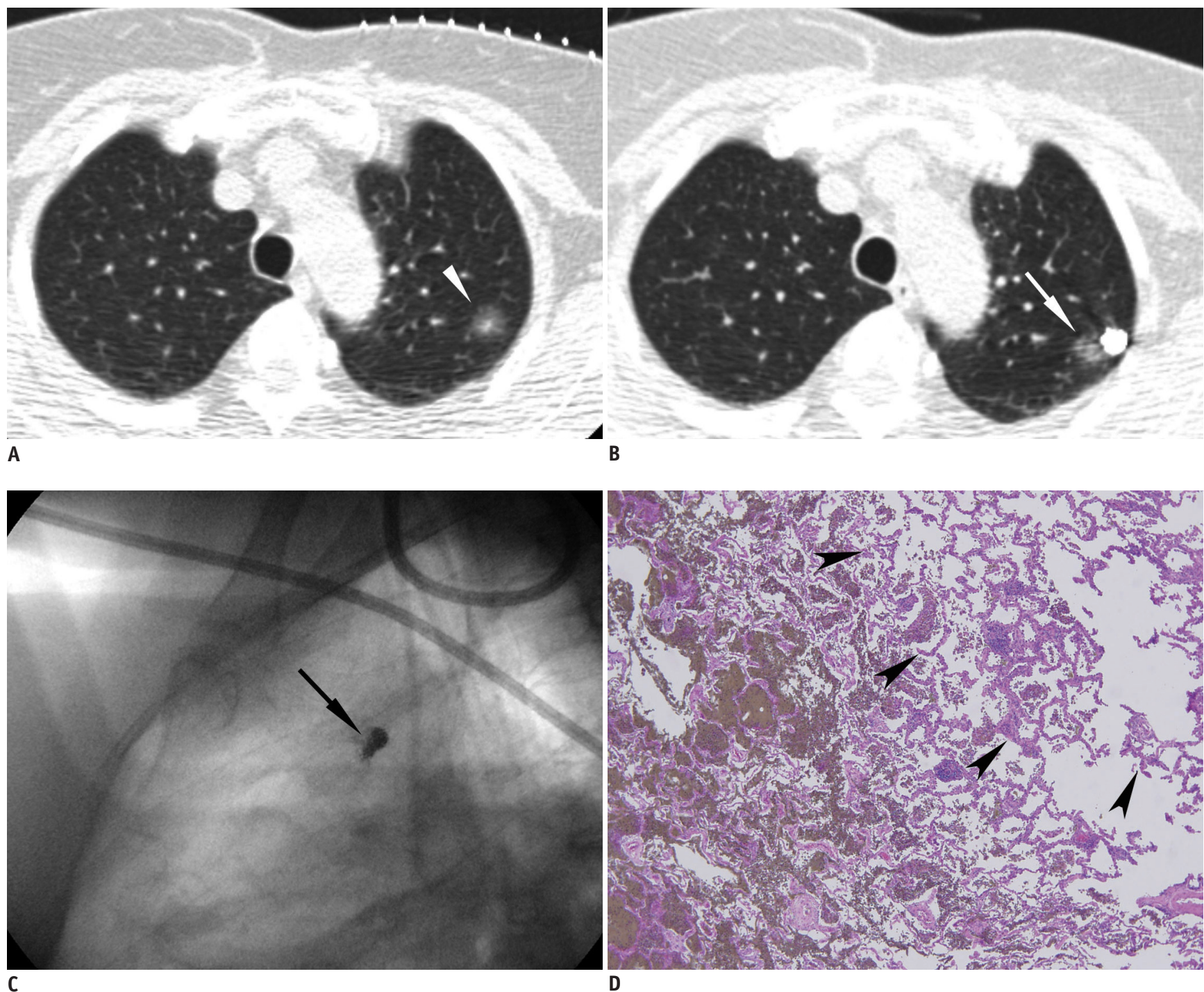

Fig. 1. Percutaneous localization of part-solid nodule in left upper lobe using barium sulfate in 68-year-old woman.

A. Appropriate barium injection site and needle route were determined using pre-procedural CT. Note part-solid nodule (arrowhead) in left upper lobe and radiopaque grid made of metallic wires on chest wall. B. Barium sulfate suspension was injected adjacent to target nodule (arrow) and appears as radiopaque ball on post-procedural CT image. C. Intraoperative fluoroscopic image shows radiopaque barium ball (arrow) in left upper lung zone. D. Injected barium sulfate appears as brownish materials dispersed within alveolar space. Note nodule seen as part-solid nodule on CT (arrowheads) near injected barium materials. There are acute inflammatory reactions containing many neutrophils, histiocytes and some eosinophils around injected barium material (original magnification, x 200; Hematoxylin-Eosin stain). Target nodule was confirmed as adenocarcinoma-in-situ, pathologically.

was encroached with barium in one patient, closely located within $0.1 \mathrm{~mm}$ in 3 , and separated from barium materials within $3 \mathrm{~mm}$ in the remaining 6 patients. In all patients, there were acute inflammations infiltrated by neutrophils and histocytes and a considerable amount of eosinophils in the barium-containing sites (Figs. 1D, 2D, 3D). However, these inflammations did not hinder the pathologic diagnosis of the nodules.

\section{DISCUSSION}

Video-assisted thoracoscopic surgery has been widely accepted as a useful and minimally invasive procedure for the resection of peripheral small or faint, albeit clinically significant lesions. However, as such lesions may not be palpable or visible during the operation (7), a conversion from VATS to thoracotomy is sometimes prescribed after failure to localize these lesions (14). Therefore, it is crucial to accurately localize such lesions prior to VATS, especially in the case of small nodules not near the pleural surface or 

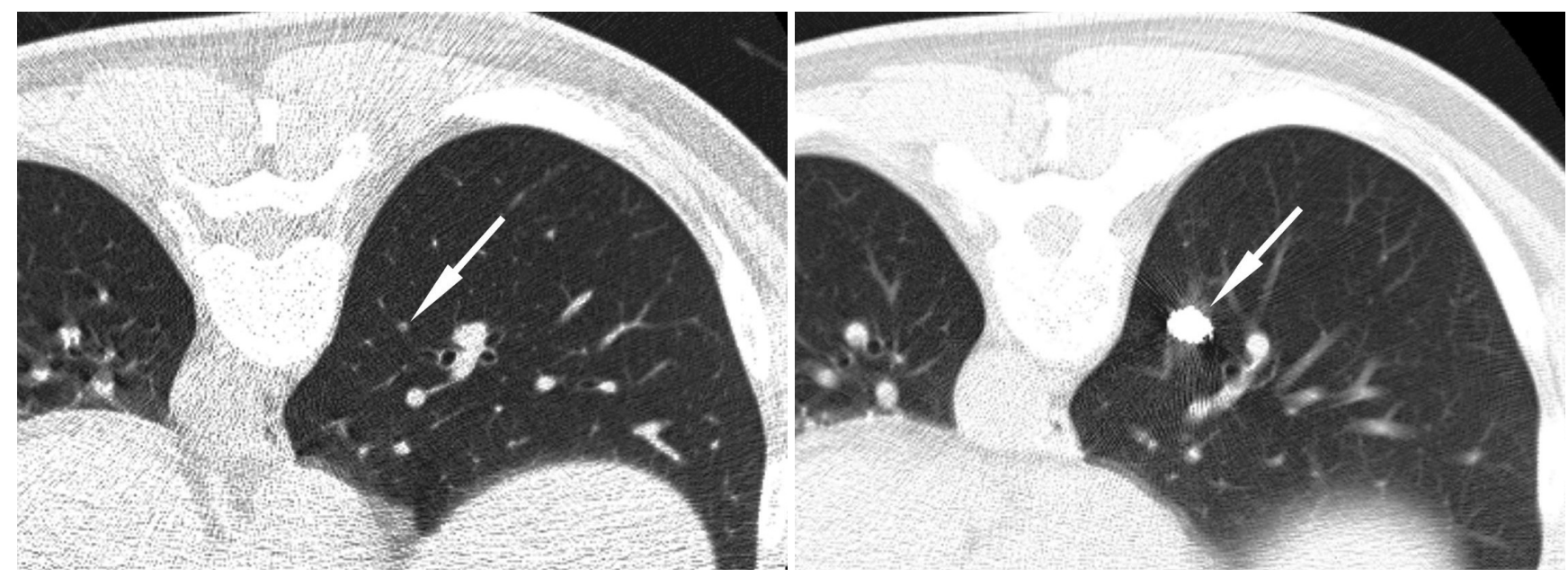

A

B
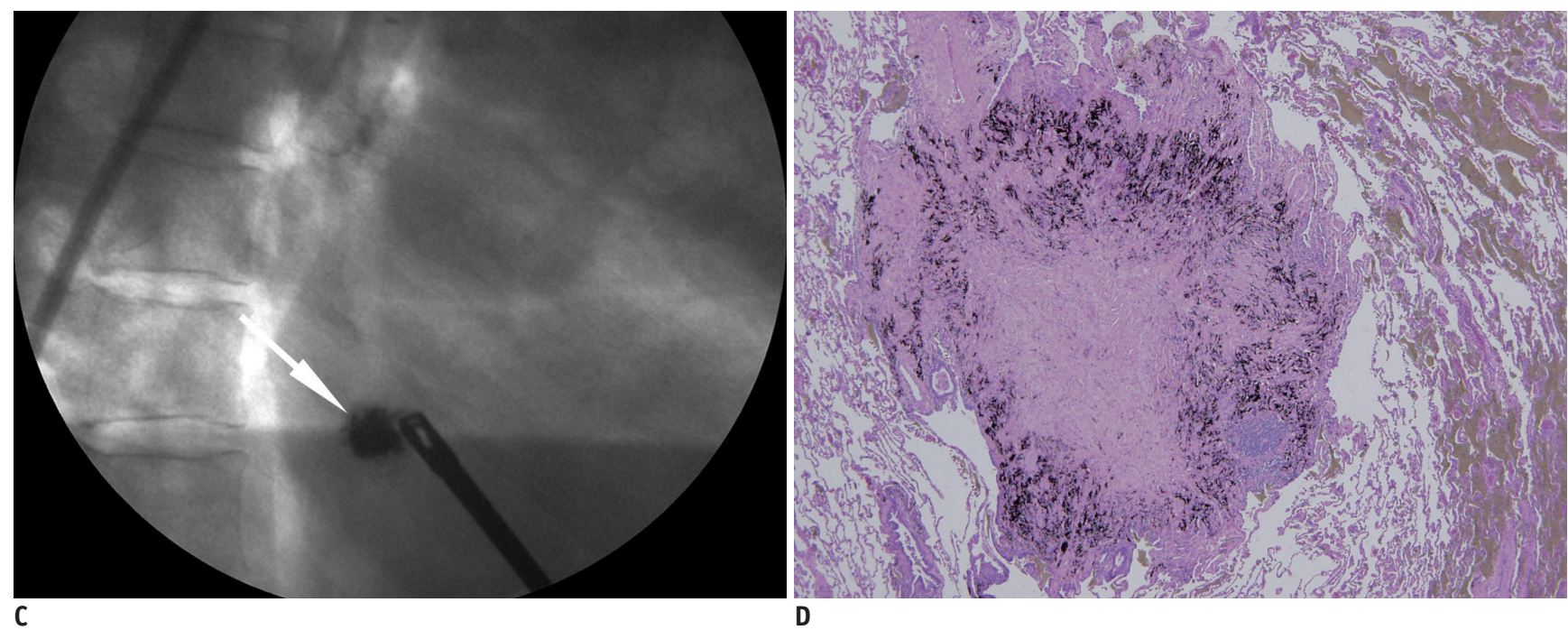

Fig. 2. Percutaneous transthoracic localization of tiny solid nodule prior to VATS using barium sulfate in 56-year-old man with medical history of colon cancer.

A. Tiny solid nodule was found on routine follow-up CT scan (arrow). B. Post-procedural CT image shows injected barium ball encroaching target nodule (arrow). C. Intraoperative fluoroscopic image shows radiopaque barium ball (arrow) in right lower lung zone. D. Brownish barium sulfate is shown around target nodule confirmed as intrapulmonary lymph node, pathologically. VATS = video-assisted thoracoscopic surgery

in faint nodules such as GGNs.

In this endeavor, several localization techniques have been introduced using either a percutaneous approach or a transbronchial approach. However, the transbronchial approach has remained technically challenging, particularly with peripherally located small nodules. Thus percutaneous localization procedures are often the main method for localization of lung nodules, particularly under the guidance of CT scans.

Various materials have been used for the percutaneous localization of nodules under CT guidance, including the injection of a methylene blue solution (15), colored collagen (16), or lipiodol (17-19), as well as the introduction of a metallic hook-wire $(7,20,21)$ or metallic microcoils $(22,23)$. They all present some problems. In the case of methylene blue, a tendency to diffuse rapidly into the surrounding lung parenchyma after dye injection was observed, and therefore, lesion localizations using methylene blue should be performed within 3 hours prior to VATS resection. This requires the simultaneous use of both $\mathrm{CT}$ and the operating room (15). Furthermore, it has been reported that up to $7 \%$ of attempts to localize nodules failed during VATS (15) due to the diffusion of dye or severe anthracosis of the visceral pleura. Lipiodol has been shown to sometimes cause aspiration into the lung, leading to damage and acute inflammations in the marking site (24). The introduction of a metallic hook wire is currently the most commonly used method for percutaneous localization 


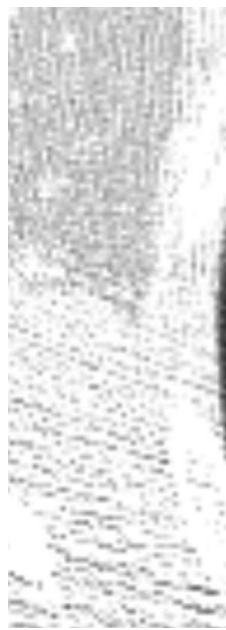

A
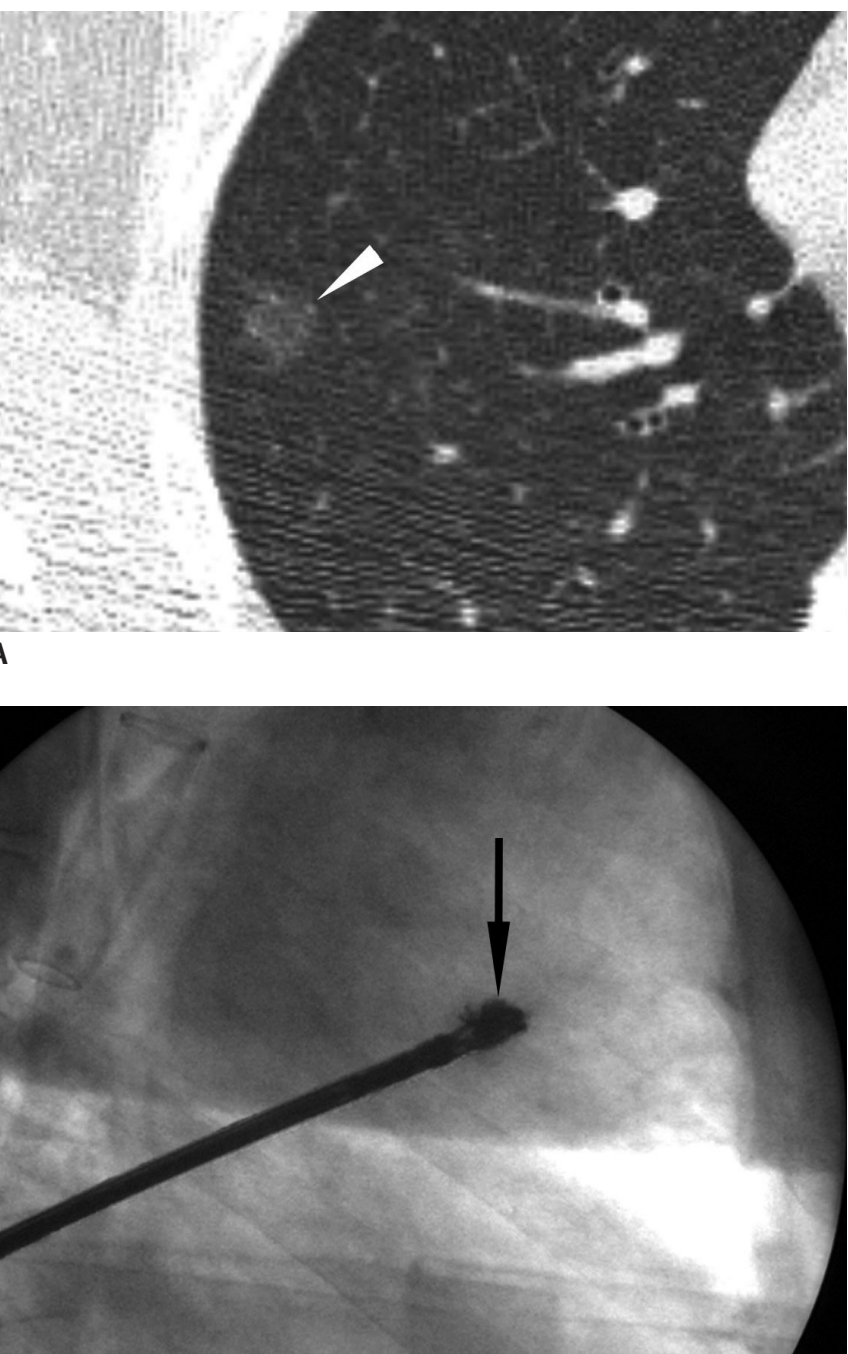

C

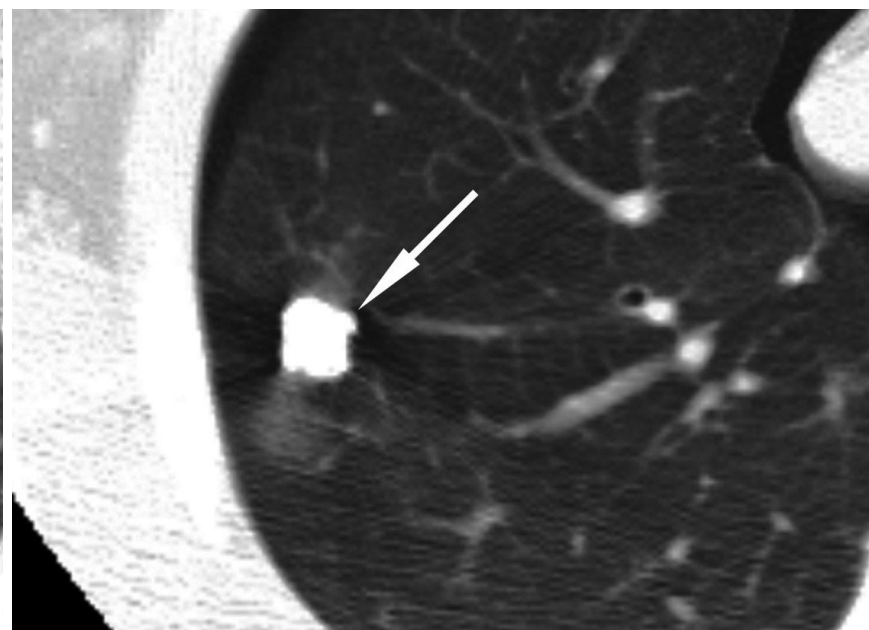

B

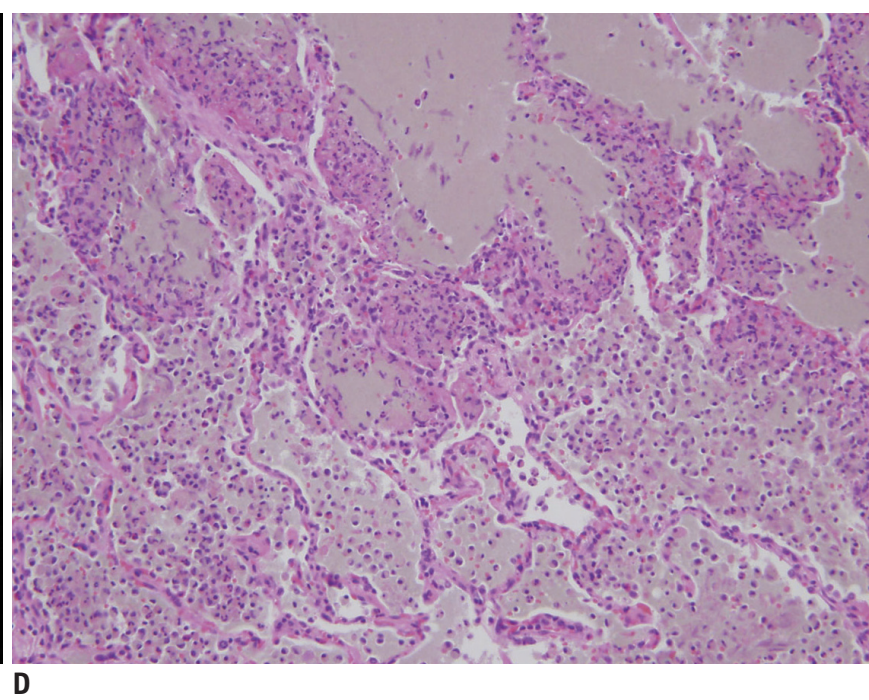

Fig. 3. Preoperative localization of pure ground-glass nodule using barium sulfate in right upper lobe found in 62-year-old man.

A. Thin-section chest CT scan shows $1 \mathrm{~cm}$ pure ground-glass nodule (arrowhead) in right upper lobe. B. Injected barium near target nodule is clearly demonstrated on post-procedural CT (arrow). Note small amount of pneumothorax which occurred during localization procedure. C. Intraoperative fluoroscopic imaging shows radiopaque barium ball (arrow) in right upper lung zone. D. Scattered brownish colored barium with acute inflammation reaction is shown. Lesion was confirmed as atypical adenomatous hyperplasia, pathologically.

of lung nodules. However, wire dislodgement occurs in up to $20 \%$ of cases (13). In addition, having a metallic wire in the chest wall can cause discomfort to the patients, and once again the simultaneous use of both CT and the operating room is necessary. Moreover, recently, massive air embolisms have been reported during the hook-wire the localization procedure $(8,9)$, although every percutaneous localization technique has a potential risk of air embolism. In another lesion localization method, Lizza et al. (22) adapted a micro-coil $15-\mathrm{mm}$ long and 5-mm in diameter. However, this is even larger than the hook-wire and lung tissue damage can be even greater than the hook wire localization, possibly increasing the risk of massive air embolism. A study by Iwasaki et al. (13) reported that bronchoscopic barium localization under the guidance of $\mathrm{X}$-ray fluoroscopy is possible with lesions that are visible on fluoroscopy. In addition, as barium has been observed to remain in the body for a relatively long period of time, the loss of the lesions due to dye diffusion can be avoided (25). This, in turn, can lead to flexible scheduling of the operation room and CT as there is no need for simultaneous use. As for complications, we observed symptomatic pneumothorax requiring air aspiration in only 1 of $10(10 \%)$ patients, with no significant consequences.

Several studies have reported that barium provoked a mild acute inflammatory and edematous reaction within hours followed by bronchopneumonia, and eventually granulomatous inflammation and fibrosis over succeeding 
days and months $(26,27)$. Yet according to an animal experiment (28) and previous clinical studies (10-13), a small volume of barium instillation did not show significant pulmonary parenchymal damage and is considered to be safe $(10-13,28)$. We believe that the very small amount of barium sulfate used in our study is does not pose that risk. The injected barium material may have caused focal inflammation but it did not hinder the histopathologic diagnosis of the target nodule in our study. Indeed, several previous studies dealing with barium localization also showed that injected barium did not cause any difficulty in the pathologic diagnosis of the target nodules (10-13). However, we would like to stress is that in the case of GGNs or potential inflammatory lesions, care should be taken to avoid directly injecting the barium into the target nodule as this may make the pathologic diagnosis of the target nodule difficult due to acute inflammation associated with barium materials.

Our study had several limitations. First, we included only a small number of patients. Second, we did not directly compare barium localization to other localization methods. We expect that further comparative studies with a prospective randomized design can give a more definitive answer on which localization method is the best for the purpose of lung nodule localization prior to VATS.

In conclusion, CT-guided percutaneous transthoracic barium localization can be an effective, safe, and convenient pre-operative localization procedure with minimal invasiveness for non-palpable small or ground-glass nodules prior to VATS.

\section{REFERENCES}

1. Pastorino U. Lung cancer screening. Br J Cancer 2010;102:1681-1686

2. Swensen SJ, Jett JR, Hartman TE, Midthun DE, Mandrekar SJ, Hillman SL, et al. CT screening for lung cancer: five-year prospective experience. Radiology 2005;235:259-265

3. Henschke CI, McCauley DI, Yankelevitz DF, Naidich DP, McGuinness G, Miettinen OS, et al. Early Lung Cancer Action Project: overall design and findings from baseline screening. Lancet 1999;354:99-105

4. Henschke CI, Yankelevitz DF, Mirtcheva R, McGuinness G, McCauley D, Miettinen OS; ELCAP Group. CT screening for lung cancer: frequency and significance of part-solid and nonsolid nodules. AJR Am J Roentgenol 2002;178:1053-1057

5. Congregado M, Merchan RJ, Gallardo G, Ayarra J, Loscertales J. Video-assisted thoracic surgery (VATS) lobectomy: 13 years' experience. Surg Endosc 2008;22:1852-1857
6. Karasaki T, Nakajima J, Murakawa T, Fukami T, Yoshida Y, Kusakabe M, et al. Video-assisted thoracic surgery lobectomy preserves more latissimus dorsi muscle than conventional surgery. Interact Cardiovasc Thorac Surg 2009;8:316-319; discussion 319-320

7. Chen S, Zhou J, Zhang J, Hu H, Luo X, Zhang Y, et al. Videoassisted thoracoscopic solitary pulmonary nodule resection after CT-guided hookwire localization: 43 cases report and literature review. Surg Endosc 2011;25:1723-1729

8. Horan TA, Pinheiro PM, Araújo LM, Santiago FF, Rodrigues MR. Massive gas embolism during pulmonary nodule hook wire localization. Ann Thorac Surg 2002;73:1647-1649

9. Iguchi T, Yoshioka T, Muro M, Miyasho K, Inoue D, Hiraki T, et al. Systemic air embolism during preoperative pulmonary marking with a short hook wire and suture system under CT fluoroscopy guidance. Jpn J Radiol 2009;27:385-388

10. Kobayashi T, Kaneko M, Kondo H, Nakayama H, Asamura H, Tsuchiya R, et al. CT-guided bronchoscopic barium marking for resection of a fluoroscopically invisible peripheral pulmonary lesion. Jpn J Clin Oncol 1997;27:204-205

11. Okumura T, Kondo H, Suzuki K, Asamura H, Kobayashi T, Kaneko M, et al. Fluoroscopy-assisted thoracoscopic surgery after computed tomography-guided bronchoscopic barium marking. Ann Thorac Surg 2001;71:439-442

12. Asano F, Shindoh J, Shigemitsu K, Miya K, Abe T, Horiba $M$, et al. Ultrathin bronchoscopic barium marking with virtual bronchoscopic navigation for fluoroscopy-assisted thoracoscopic surgery. Chest 2004;126:1687-1693

13. Iwasaki Y, Nagata K, Yuba T, Hosogi S, Kohno K, Ohsugi S, et al. Fluoroscopy-guided barium marking for localizing small pulmonary lesions before video-assisted thoracic surgery. Respir Med 2005;99:285-289

14. Suzuki K, Nagai K, Yoshida J, Ohmatsu H, Takahashi K, Nishimura $M$, et al. Video-assisted thoracoscopic surgery for small indeterminate pulmonary nodules: indications for preoperative marking. Chest 1999;115:563-568

15. Wang YZ, Boudreaux JP, Dowling A, Woltering EA. Percutaneous localisation of pulmonary nodules prior to video-assisted thoracoscopic surgery using methylene blue and TC-99. Eur J Cardiothorac Surg 2010;37:237-238

16. Nomori $\mathrm{H}$, Horio $\mathrm{H}$. Colored collagen is a long-lasting point marker for small pulmonary nodules in thoracoscopic operations. Ann Thorac Surg 1996;61:1070-1073

17. Kwon WJ, Kim HJ, Jeong YJ, Lee CH, Kim KI, Kim YD, et al. Direct lipiodol injection used for a radio-opaque lung marker: stability and histopathologic effects. Exp Lung Res 2011;37:310-317

18. Kawanaka K, Nomori H, Mori T, Ikeda K, Ikeda 0, Tomiguchi $S$, et al. Marking of small pulmonary nodules before thoracoscopic resection: injection of lipiodol under CTfluoroscopic guidance. Acad Radiol 2009;16:39-45

19. Moon SW, Wang YP, Jo KH, Kwack MS, Kim SW, Kwon OK, et al. Fluoroscopy-aided thoracoscopic resection of pulmonary nodule localized with contrast media. Ann Thorac Surg 1999;68:1815-1820 
20. Federici S, Ratta A, Mordenti M, Domenichell V, Pelusi $G$, Italiano F, et al. Successful thoracoscopic resection of pulmonary metastasis less than $1 \mathrm{~cm}$ in size utilizing preoperative CT-guided wire localization. J Laparoendosc Adv Surg Tech A 2009;19 Suppl 1:S171-S173

21. Chen YR, Yeow KM, Lee JY, Su IH, Chu SY, Lee CH, et al. CTguided hook wire localization of subpleural lung lesions for video-assisted thoracoscopic surgery (VATS). J Formos Med Assoc 2007;106:911-918

22. Lizza N, Eucher P, Haxhe JP, De Wispelaere JF, Johnson PM, Delaunois $\mathrm{L}$. Thoracoscopic resection of pulmonary nodules after computed tomographic-guided coil labeling. Ann Thorac Surg 2001;71:986-988

23. Mayo JR, Clifton JC, Powell TI, English JC, Evans KG, Yee J, et al. Lung nodules: CT-guided placement of microcoils to direct video-assisted thoracoscopic surgical resection. Radiology 2009;250:576-585
24. Felton WL. The reaction of pulmonary tissue to lipiodol. $J$ Thorac Surg 1953;25:530-542

25. Erickson LM, Shaw D, MacDonald FR. Prolonged barium retention in the lung following bronchography. Radiology 1979;130:635-636

26. Gray C, Sivaloganathan S, Simpkins KC. Aspiration of highdensity barium contrast medium causing acute pulmonary inflammation--report of two fatal cases in elderly women with disordered swallowing. Clin Radiol 1989;40:397-400

27. Ginai AZ, ten Kate FJ, ten Berg RG, Hoornstra K. Experimental evaluation of various available contrast agents for use in the upper gastrointestinal tract in case of suspected leakage. Effects on lungs. Br J Radiol 1984;57:895-901

28. Thompson IM, Whittlesey GC, Slovis TL, Chang CH, Cullen ML, Philippart AI, et al. Evaluation of contrast media for bronchography. Pediatr Radiol 1997;27:598-605 